\title{
Ser childfree en México: narrativas personales de quienes no desean ser madres o padres y su negociación con los estigmas sociales
}

\section{Being Childfree in Mexico: Personal Narratives of People Choosing not to Become Parents and How They Cope with Social Stigmas}

Recibido: 5 de abril de

2021

Aceptado: 24 de agosto de 2021

Publicado: 20 de

septiembre de 2021

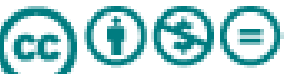

BY NC ND
Esta obra está protegida bajo una Licencia Creative Commons Atribución-NoComercialSinDerivadas 4.0 Internacional (CC BY-NC-ND 4.0)

\section{Resumen}

El presente artículo explora la elección childfree en México e indaga particularmente el impacto de los estigmas sociales hacia las personas que deciden rechazar para sí la maternidad/paternidad. Luego de extraer las cualidades y roles normativos de la masculinidad y feminidad de los discursos hegemónicos en la sociedad mexicana, a partir del análisis interpretativo de encuestas en línea y entrevistas en profundidad, se develan las narrativas que emplearon las personas que se identifican a sí mismas como childfree para negociar con los estigmas sociales y defender su decisión. El alcance de este estudio exploratorio es limitado, pero sienta bases para la construcción de un

CÓMO CITAR: Mandujano-Salazar, Yunuen. (2021). Ser childfree en México: narrativas personales de quienes no desean ser madres o padres y su negociación con los estigmas sociales. Revista Interdisciplinaria de Estudios de Género de El Colegio de México, 7, e756. doi: http://dx.doi.org/10.24201/reg.v7i1.756

http://estudiosdegenero.colmex.mx 
panorama del fenómeno en México y visibiliza diversas problemáticas que requieren analizarse en profundidad. Asimismo, a diferencia de la tendencia de estudios sobre childfree alrededor del mundo, en este se incluyeron no sólo a mujeres, sino también a varones, buscando las similitudes y diferencias entre los estigmas que perciben y sus implicaciones en círculos privados y laborales. Los hallazgos nutren el debate relacionado con identidades de género en América Latina.

Palabras clave: maternidad; paternidad; identidad de género; narrativas personales; México.

\begin{abstract}
This article looks at the choice made by Mexican men and women to be childfree, focusing on the impact of social stigmas on people who decide not to become parents. After extracting, from hegemonic discourses, the normative aspects and roles of femininity and masculinity in Mexican society, an interpretative analysis of online surveys and in-depth interviews are used to reveal the personal narratives used by childfree people to cope with the stigmas attached to their decision. This explorative study has a limited scope but lays the foundation for an understanding of the phenomenon in Mexico, giving visibility to diverse issues for further analysis. Unlike most childfree studies around the world, this article looks at the experience of both women and men, seeking similarities and differences among the stigmas they face and the implications in their private and professional lives. The findings add to the gender identity debate in the Latin America.

Key words: childfree; motherhood; fatherhood; gender identity; personal narratives; Mexico
\end{abstract}

\title{
Introducción
}

Los sistemas sexo-género han dividido a los seres humanos en dos grandes categorías — hombres y mujeres - se les asignan, por medio de discursos hegemónicos, determinados papeles públicos y privados, rasgos de personalidad socialmente esperados y se establecen las trayectorias de vida 
que deben seguir. Sin embargo, desde la última mitad del siglo XX, son cada vez más los debates sobre temáticas de género que han pugnado por deconstruir esas ideologías binarias y deterministas. Importantes intelectuales han desenmascarado las ideologías y los sistemas de control social que se encuentran detrás de las representaciones y expectativas de los sistemas sexo-género en las sociedades contemporáneas (Butler, 1990, 2011; de Beauvoir, 2014; Fraser, 1997; Ginsburg y Rapp, 1991; Gonzalez-Barrientos y Napolitano, 2015; Jones, 1984). Esto, junto con los movimientos de millones de activistas alrededor del mundo, ha permitido que la diversidad de género y de preferencias sexuales vayan, poco a poco, integrándose como parte de los imaginarios sociales tolerados — aunque no plenamente aceptados - en cada vez más sociedades.

La elección childfree, o "libre de hijos", es otro fenómeno que está relacionado con la deconstrucción de los sistemas sexo-género y los cambios en las trayectorias de vida propios de las sociedades contemporáneas. Ser childfree implica la renuncia personal y voluntaria a la maternidad o paternidad. Sin embargo, al ser estas unas de las funciones biológico-sociales más naturalizadas - atribuibles a la gran mayoría de los individuos, relacionadas tradicionalmente con ritos de paso a la adultez, con roles deseables de la masculinidad y feminidad hegemónicas, pero, sobre todo con políticas de reproducción que son claves para el sostenimiento del sistema económico y de producción - renunciar voluntariamente a ellas sigue generando cuestionamientos y estigmas sociales, con los cuales las personas childfree deben negociar cotidianamente.

Sumándose al creciente debate sobre la tendencia childfree alrededor del mundo, en el marco de los estudios de género, este artículo presenta los resultados de un estudio exploratorio enfocado en analizar las narrativas de personas mexicanas legalmente adultas que se declaran childfree. Se ha decidido utilizar este término en inglés puesto que el interés dentro de la presente investigación está centrado en quienes ejercen su agencia al elegir no ser madres o padres y la inclusión de la palabra inglesa free (libre) es la principal razón por las que las personas entrevistadas expresaron preferir esa etiqueta para hacer manifiesta su decisión. El objetivo es explorar, a partir de encuestas y entrevistas en profundidad, las formas en las cuales, en una sociedad patriarcal y pronatalista como la mexicana, las personas perciben los estigmas sociales 
y negocian con los discursos hegemónicos de género para construirse narrativamente cuando deciden que no desean asumir esos roles en su vida. Se busca visibilizar a mujeres y hombres childfree de México y comenzar a comprender las formas en que conforman el sentido de su identidad social cuando se encuentran fuera de los papeles normativos, cómo negocian con los discursos hegemónicos, y si existen diferencias entre varones y mujeres en dicha negociación. Es importante recalcar que aquí se estará hablando de la elección personal childfree y no de un movimiento antinatalista.

Primeramente, se presenta una reconstrucción de los discursos dominantes acerca de la masculinidad y la feminidad en México, para luego problematizar la elección childfree como fenómeno de estudio y argumentar la relevancia de analizarla con un enfoque en la construcción identitaria de los individuos frente a los discursos dominantes de género y los estigmas particulares de cada sociedad. En el apartado metodológico se construye un panorama acerca de las características de las personas childfree en México, de acuerdo con las encuestas realizadas. Posteriormente, se analizan las narrativas de los informantes para dilucidar los estigmas que identifican y su negociación con ellos. Por último, se plantean las reflexiones finales acerca de las formas en que los individuos debaten con los discursos hegemónicos y se construyen a sí mismos.

\section{De deberes y bendiciones: los discursos hegemónicos de género en México}

México es un país con profundas diferencias étnicas, culturales y socioeconómicas. No obstante, la moral y los valores católicos han influenciado por siglos los discursos hegemónicos de género, aun cuando la afiliación a tal religión ha ido disminuyendo en los últimos años ${ }^{1}$. La aceptación social de las relaciones sexuales limitada a parejas heterosexuales y con el objetivo primordial de la procreación ha sido uno de los principios ideológicos reguladores de la sexualidad que ha nutrido los discursos que siguen promoviendo la maternidad y paternidad como ritos naturales de paso a la adultez, y claves en la caracterización de los roles tradicionales de género: varones que se espera asuman los roles de padres, esposos, protectores y proveedores económicos; y

\footnotetext{
${ }^{1}$ En 2020, de acuerdo con el Censo de Población y Vivienda, el $77.7 \%$ de la población declaró ser católica, a diferencia del 82.7\% en 2010 (Díaz Domínguez, 2021). 
mujeres a quienes se les han asignado como esenciales los roles de esposas, madres, educadoras y proveedoras de cuidados (Instituto Nacional de las Mujeres, 2007). Históricamente, la masculinidad hegemónica mexicana ha sido relacionada con características de fuerza física y mental, inteligencia, valor, poder, habilidad para obtener resultados, agresividad y homofobia; mientras que la feminidad hegemónica ha dictado que las mujeres deben ser dóciles, modestas, compasivas, atentas a las necesidades de otros y estoicas (Aguilar Montes de Oca, Medina, González-Arratia López-Fuentes y González Escobar, 2013).

Aunque, en la década de 1910, durante la Revolución Mexicana, figuras femeninas aparecieron como parte de los grupos beligerantes - las soldaderas - durante las siguientes dos décadas, luego de que la guerra civil concluyó y cuando los líderes mexicanos estaban reconstruyendo el Estado, estas mujeres con su característica valentía, fuerza e independencia fueron representadas en los discursos dominantes como marimachas que amenazaban a la nación (Ruiz-Alfaro, 2018). De hecho, en el Artículo 15 de la Ley de Matrimonio Civil de la Constitución de 1917 quedaron asentadas las características esperadas de cada género - que habrían de nutrir estereotipos- y los roles esencialmente relacionados a la reproducción:

Que éste es el único medio moral de fundar la familia, de conservar la especie y de suplir las imperfecciones del individuo que no puede bastarse á si mismo para llegar á la perfección del género humano. Que éste no existe en la persona sola sino en la dualidad conyugal. [...] Que el hombre cuyas dotes sexuales son principalmente el valor y la fuerza, debe dar, y dará á la mujer, protección, alimento [...] Que la mujer, cuyas principales dotes son la abnegación, la belleza, la compasión, la perspicacia y la ternura, debe dar y dará al marido obediencia [...] Que la sociedad bendice, considera y, alaba á los buenos padres (Instituto Nacional de Estudios Históricos de las Revoluciones de México, 2015, pp. 3-4).

Durante la década de 1940, mientras México vivía un resurgimiento del nacionalismo en el contexto de la Segunda Guerra Mundial, los machos comenzaron a ser promovidos en el cine como los mexicanos alfa, a través de estrellas como Pedro Infante o Jorge Negrete. Macho se convirtió en una noción que envolvía los elementos contradictorios de violencia, exceso de 
bebida, infidelidad, valor, romanticismo y devoción a la nación (Berghegger, 2009). Al mismo tiempo, el gobierno comenzó a auspiciar una imagen de la mujer moderna que involucraba la feminidad tradicional añadiendo elementos que eran necesarios para los intereses políticos y económicos de las élites. Se esperaba que las mujeres modernas mexicanas fueran madres y cuidaran de sus hijos, esposos y familias, pero también que ayudaran a la nación y apoyaran la economía del hogar incorporándose a la fuerza de trabajo. La maternidad y el sacrificio de las mujeres para cumplir con los papeles doméstico y público, su devoción y patriotismo se reforzaban en la propaganda de este tiempo como pruebas de feminidad (Rankin, 2011).

Tal como analizó Cardozo-Freeman (1975), incluso en los juegos y canciones tradicionales infantiles — de fuerte influencia católica - que se transmitían de generación en generación al menos hasta la década de 1970, se inculcaba a las mujeres que debían ser sumisas, sacrificarse y ser estoicas ante las múltiples restricciones a las que eran sometidas. Las mujeres debían mantenerse vírgenes hasta el matrimonio y no disfrutar de su sexualidad, sino verla como una obligación al igual que la maternidad, - que era una forma de control por parte del esposo para demostrar tanto su virilidad como para asegurar la fidelidad de $s u$ mujer.

No obstante, ya para finales de la década de 1960, el contexto económico, el mercado laboral y los discursos políticos y sociales influenciados por las tendencias mundiales — principalmente norteamericanas, como los movimientos feministas y la revolución sexual- comenzaron a impulsar cambios en el modelo de familia de la sociedad urbana en México. Conforme las mujeres comenzaron a tener acceso a la educación superior y a mejores empleos y sueldos, obtuvieron algo de poder para negociar su estatus con los varones, quienes debieron adaptar sus expectativas acerca de lo que una pareja femenina haría por ellos. Como resultado de los cambios en la distribución de poder entre sexos y la constante lucha de las mujeres por la equidad en el ámbito privado, en las últimas dos décadas, la tasa de matrimonios legales ha decrecido, mientras los divorcios y las uniones libres han aumentado; de acuerdo con las estadísticas de 2019 , sólo el $38 \%$ de la población de quince años y más estaba casada, a diferencia del 49\% en el año 2000 (Instituto Nacional de Estadística y Geografía, 2021). 
A pesar de esto, el patriarcado y la heteronormatividad persisten en el país, modificando el tono de los discursos, pero prevaleciendo los roles asignados a los hombres y a las mujeres para ser socialmente reconocidos. En el caso de éstas, tener hijos es, de acuerdo con la sociedad mexicana, una bendición que da sentido a la vida de la mujer; por tanto, un aspecto clave para la construcción de la identidad femenina (Aguilar Montes de Oca, et al., 2013). La figura de la madre sigue siendo una de las más valoradas y el discurso de la entrega incondicional y desinteresada de las madres mexicanas inunda la cultura nacional (Schell, 2012). Así, aun cuando las mujeres mexicanas han conseguido mayor capacidad económica y, hasta cierto punto, pueden escapar del estigma social por no estar legalmente casadas, la sociedad sigue presionándolas para que sean madres y enfrenten estoicamente los sacrificios que implica la maternidad como algo que debe darles felicidad y significar la plenitud de su feminidad (Ávila González, 2005; García Delgado y Riquelme Vigueras, 2017). Esto se ha traducido en una elevada proporción de mujeres que llevan la maternidad sin una pareja -incluidas mujeres viudas, divorciadas, separadas o solteras. En 2014, 33\% de las mujeres de quince a cincuenta y cuatro años que eran madres eran solteras bajo tal definición (Instituto Nacional de Estadística y Geografía, 2018).

Por otro lado, aún existe la expectativa de que los hombres demuestren su virilidad siendo autosuficientes, teniendo una pareja femenina, procreando, siendo cabezas de familia y asumiendo la mayor carga de proveeduría (Aguilar Montes de Oca, et al., 2013; Gutmann, 2000). Particularmente, la procreación — si bien ya no tiene que ser numerosa - sigue siendo, entre la mayoría de los estratos sociales, la forma de acceder a una posición social de mayor reconocimiento como hombres: "Los hijos, al parecer, son una marca de distinción en las relaciones entre los varones, pues un hombre debe ser capaz de responsabilizarse de sus hijos, hecho que marca la diferencia que separa y jerarquiza a los varones" (Rojas Martínez, 2012, pp. 84-85).

Por lo tanto, a pesar de que la sociedad mexicana ha cambiado, todavía es una expectativa dominante, incluso entre jóvenes, que hombres y mujeres se conviertan en padres y madres y demuestren las características atribuidas a cada género dentro de esos roles (Hernández Herrera, 2019). 


\section{La reproducción, la elección childfree y los estigmas}

Foucault (2005), a partir de sus postulados sobre el biopoder y la biopolítica, discernió sobre cómo el cuerpo de los individuos y las funciones del mismo - todo lo biológico - han sido de interés público por siglos. Por medio de discursos sobre el cuerpo humano y sus funciones, con particular vehemencia sobre aquello relacionado con lo sexual, se ha controlado a los seres humanos de distintas maneras para beneficio de los sectores que ejercen el mayor poder en una sociedad.

Si bien, la decisión de un individuo sobre su vida reproductiva se supondría un tema personal, la reproducción es, sin duda, un tema de importancia para la supervivencia y el desarrollo de las sociedades; tanto un desborde como una contracción en exceso de la tasa de fecundidad tiene un impacto en distintos ámbitos, principalmente en la producción y el sistema económico en general. Por tanto, ha sido una de las principales funciones biológicas humanas que los Estados han manipulado por medio de políticas de reproducción que incluyen tanto el acceso o restricción a servicios médicos, como estrategias discursivas implementadas con ayuda de los sistemas educativos, instituciones religiosas, medios de comunicación y las mismas instituciones de salud y gubernamentales (Ginsburg y Rapp, 1991). Morgan y Roberts (2012) consideran que la gobernanza de la reproducción implica todos los mecanismos, tales como controles legislativos, ideas morales, discursos éticos y coerción directa, que utilizan las instituciones para producir, monitorear y controlar los comportamientos y prácticas reproductivas de la población.

En las sociedades que se dicen democráticas, los controles legislativos y las estrategias discursivas son las más evidentes para estos efectos. Debido a que la tasa de fecundidad de reemplazo ha sido determinada por los demógrafos de la modernidad en 2.1 hijos por mujer (MacInnes y Pérez Díaz, 2009), los discursos hegemónicos han mantenido naturalizadas la maternidad y la paternidad, poniéndolas como funciones esenciales de los individuos para que continúen siendo primordiales en la construcción identitaria de las personas. Así, la adultez y la identidad de género siguen estando fuertemente ligadas a la reproducción y al ejercicio de los roles de madre o padre, interfiriendo en la construcción positiva del adulto que decide o se ve en la circunstancia de no cumplir con ese papel (Mandujano-Salazar, 2019). 
No obstante, en medio de distintas crisis económicas, ambientales y de seguridad pública, ante la precarización laboral y, sobre todo, ante la relevancia que ha adquirido el debate feminista invitando a las mujeres a decidir sobre su cuerpo y sus trayectorias de $v^{2}{ }^{2}{ }^{2}$, se está presentando una tendencia ascendente de personas que están decidiendo no ser padres o madres. Las motivaciones, problemáticas que enfrentan y consecuencias relacionadas con este fenómeno son transversales a múltiples campos del conocimiento. La renuncia voluntaria a la paternidad o maternidad se ha convertido en un tema social, cultural, económico y político conforme se vuelve más notorio. Por ello, los estudios sobre personas childfree en diferentes países han ido incrementándose en los últimos años, poniendo sobre la mesa de discusión distintas aristas.

Uno de esos debates ha sido el término más adecuado para identificar a estas personas: voluntary childless (voluntariamente sin hijos), childless by choice (sin hijos por elección), childfree (libre de hijos) o, en el caso específico de mujeres, NoMo (No mothers, no madres). Hay quienes, como Ávila (2005), cuestionan la utilización del concepto de elección, al momento de decidir tener o no hijos, debido a que éste sugiere una igualdad de oportunidades para seleccionar libremente entre las alternativas posibles, lo que deja fuera a las relaciones de poder y las estructuras que, a fin de cuentas, restringen las posibles elecciones de las personas. No obstante, debido a las connotaciones que tiene el sufijo less (menos o sin), recientemente tanto en la academia como entre las personas que toman la decisión de no tener hijos, se prefiere el término childfree, pues pone énfasis en la libertad y agencia de los individuos y los distingue de quienes desean tener hijos, pero, por diversas circunstancias no pueden tenerlos (Bartlett, 1996).

A pesar del intento por asumir la decisión de manera positiva - como una forma de agencia y libertad - el estigma que sufren las personas childfree parece ser persistente. A diferencia de un estereotipo -el cual implica ideas fijas y simplificadas, que pueden ser positivas o negativas, asociadas a una persona o grupo - el estigma es una marca, condición o estatus que lleva a la devaluación del individuo y esa condición es formada en un contexto cultural específico

\footnotetext{
${ }^{2}$ Este debate feminista se puede identificar, por ejemplo, en Simone de Beauvoir (2014) quien llamó a las mujeres a reclamar su derecho a construir su vida sin los determinismos de la cultura o en Judith Butler $(1990,2011)$ quien puso énfasis en la performatividad del género, también en las activistas y académicas feministas que han pugnado porque se reconozca el derecho de la mujer tanto a abortar, como a tener hijos sin tener una pareja masculina, por ejemplo, Petchesky (1990) y Robertson (1994).
} 
(Goffman, 2006; Link y Phelan, 2001). En el caso de las personas childfree, el estigma va de la mano con los discursos hegemónicos de género de cada contexto social.

Por ejemplo, en el caso de la sociedad japonesa, en donde existe una crisis demográfica y la maternidad y paternidad siguen fuertemente ligadas al matrimonio, el estigma va relacionado a la soltería. En los años de cambio de siglo, el término solteros parásitos se utilizó ampliamente tanto en los medios, como en las instituciones gubernamentales y la academia para referirse a las personas mayores de veinte años que vivían con sus padres (Nishi y Kan, 2006; Yamada, 1999). Los estigmas han sido particularmente notorios hacia las mujeres solteras que no son madres llamándoles, incluso, loser dogs - resaltando el contenido de derrota y falta de compromiso con la nación por, supuestamente, preferir la realización profesional a ajustarse a las trayectorias de vida socialmente esperadas (Dales, 2014, 2015; Mandujano-Salazar, 2017, 2019; Rosenberger, 2007). En Estados Unidos, en donde las tasas de natalidad están decreciendo, los estudios tienden a enfocarse en las mujeres, encontrando que la elección childfree parece relativamente más común entre quienes poseen un mayor nivel educativo o un estatus económico medio o alto y que se ha propagado el estigma de que son egoístas, que odian a la humanidad o a los niños, que quieren acabar con la especie (Chancey y Dumais, 2009; Gillespie, 2003; McCabe y Sumerau, 2018; Peterson, 2014). Un estudio sobre mujeres israelíes que no son madres encontró que aquellas que no lo son por decisión, enfrentan aislamiento social derivado de visiones negativas generalizadas sobre su decisión (Yeshua-Katz, 2018). Otra investigación, centrada en la sociedad rusa, encontró que más de la mitad de las personas encuestadas consideró que la tendencia childfree era una amenaza para Rusia (Salyakhieva y Saveleva, 2017).

Tal parece que las personas que no cumplen con la expectativa de reproducirse — sobre todo si biológicamente no tienen impedimento - son propensas a sufrir estigma social y a ser condenadas explícita o implícitamente desde los discursos hegemónicos de género. Es en este contexto en el que se localiza el presente estudio. Basta hacer una búsqueda en las redes sociales para encontrar un creciente número de grupos y blogs que reúnen a personas mexicanas que se declaran childfree y expresan sus quejas acerca de las dificultades que su decisión les ha planteado. Aun así, las motivaciones de estas personas para renunciar a la maternidad o paternidad y los problemas a los que se enfrentan - ya sea en su vida privada, profesional o 
social - apenas comienzan a ser analizados en la academia mexicana. Algunos estudios han analizado las motivaciones y experiencias de algunas mujeres mexicanas que decidieron no ser madres (Ávila González, 2005; García Delgado y Riquelme Vigueras, 2017; Quintal López, 2002). Mientras que otro comparó las narrativas personales de varones y mujeres cisgénero solteros y solteras childfree en México y Japón centrándose en su construcción identitaria al no cumplir con dos de los roles más naturalizados atribuidos al género en ambas sociedades (Mandujano-Salazar, 2019). Sin embargo, es relevante conocer cuál es el contenido del estigma que sufren las personas mexicanas childfree y comenzar a visibilizar las experiencias de los varones childfree, analizando si los estigmas y retos son similares en varones y mujeres, en personas casadas y solteras. Esto permitirá comenzar a entender tanto las razones detrás de la decisión individual, como los retos a los que se enfrentan las personas al asumir un estilo de vida que reta los discursos hegemónicos de la sociedad mexicana.

\section{Metodología}

Esta investigación parte de un enfoque cualitativo apoyado en la recolección de narrativas personales y el análisis interpretativo de las mismas. Se considera que la narrativa de una persona acerca de su vida es un sitio en el cual la identidad es negociada dentro del entorno en el que se localiza. Por su parte, la identidad se entiende como ideología comprendida por medio del involucramiento del individuo con el discurso, por lo cual se manifiesta en narrativas personales construidas y reconstruidas a través del curso de vida y escritas en y a través de la interacción y las prácticas sociales (Hammack, 2008). Por tanto, en este estudio se analizan las narrativas personales de informantes para comprender cómo han negociado su elección de ser childfree con los discursos sociales a los que se enfrentan cotidianamente sobre la maternidad/paternidad y los estereotipos que circulan sobre quienes no tienen hijos.

La recolección de información se llevó a cabo en dos etapas. A fin de tener más puntos de vista, se buscó incluir informantes de distintas ciudades del país, de diversos contextos familiares, así como de diferentes formaciones profesionales/laborales. Por ello, durante el 1 y el 15 de abril de 2020, se realizó una encuesta en línea en tres grupos de Facebook identificados como childfree en español para explorar tendencias etarias, de orientación sexual, de crianza e ideología 
religiosa, así como de experiencias y razones que llevaron a su decisión de renunciar a la maternidad/paternidad ${ }^{3}$. Se utilizó Google Forms, se identificó claramente el propósito de la encuesta y se solicitó la participación voluntaria de aquellos que fueran de nacionalidad mexicana, mayores de 18 años y que no tuvieran hijos biológicos ni adoptados. Se obtuvo un total de 168 respuestas; el formato permitía sólo una respuesta por correo electrónico registrado.

A partir de esta encuesta, se identificaron dieciséis informantes - ocho mujeres y ocho hombres - para realizar entrevistas en profundidad que permitieran la recolección de narrativas individuales extensas y el análisis de los discursos a los que se sienten enfrentadas las personas childfree en México. Debido a que se trató de un estudio exploratorio, se siguió un muestreo de caso crítico, seleccionando a quienes, a partir de las respuestas en la encuesta, podían proveer más información relevante para responder las preguntas de investigación (ver Tabla 1). Las entrevistas se realizaron virtualmente entre septiembre y diciembre de 2020, por medio de llamada o videollamada a través de la aplicación preferida por los entrevistados - Messenger, WhatsApp, Teams, Zoom. Con el permiso de los informantes, se grabó sólo el audio para realizar la transcripción y análisis por medio del software NVivo 12, mismo que se usó también para analizar las encuestas.

\footnotetext{
${ }^{3}$ Los grupos en donde se publicó la encuesta fueron: Sin Hijos Por Decisión, Libre de hijos - Childfree 3.0, y Sin hijos por elección, la vida childfree.
} 
Tabla 1. Informantes de entrevistas a profundidad

\begin{tabular}{|c|c|c|c|c|c|c|c|}
\hline Clave & Sexo & $\begin{array}{l}\text { Orientación } \\
\text { sexual }\end{array}$ & Edad & $\begin{array}{l}\text { Lugar de } \\
\text { residencia }\end{array}$ & $\begin{array}{l}\text { Educación } \\
\text { máxima }\end{array}$ & $\begin{array}{l}\text { Ocupación } \\
\text { actual }\end{array}$ & $\begin{array}{l}\text { Situación } \\
\text { romántico- } \\
\text { afectiva }\end{array}$ \\
\hline Inf1 & $\mathrm{M}$ & Heterosexual & 38 & CDMX & Maestría & Empleada & Soltera \\
\hline Inf2 & $\mathrm{M}$ & Heterosexual & 36 & Monterrey & Maestría & Empleada & Soltera \\
\hline Inf3 & $\mathrm{M}$ & Heterosexual & 28 & Cd. Juárez & Licenciatura & Empleada & Casada \\
\hline Inf4 & $\mathrm{M}$ & Heterosexual & 31 & Tijuana & Doctorado & Empleada & Casada \\
\hline Inf5 & $\mathrm{M}$ & Bisexual & 24 & CDMX & Licenciatura & $\begin{array}{l}\text { Negocio } \\
\text { propio }\end{array}$ & Tiene pareja \\
\hline Inf6 & $\mathrm{M}$ & Homosexual & 22 & Guadalajara & Licenciatura & Estudiante & Soltera \\
\hline Inf7 & $\mathrm{M}$ & Heterosexual & 30 & $\begin{array}{ll}\text { Edo. } & \text { de } \\
\text { México } & \end{array}$ & Maestría & Empleada & Soltera \\
\hline Inf8 & $\mathrm{M}$ & Heterosexual & 45 & $\begin{array}{ll}\text { San } & \text { Luis } \\
\text { Potosí } & \end{array}$ & Maestría & Empleada & Casada \\
\hline Inf9 & $\mathrm{H}$ & Heterosexual & 39 & CDMX & Doctorado & Empleado & Tiene pareja \\
\hline Inf10 & $\mathrm{H}$ & Heterosexual & 31 & Cd. Juárez & Licenciatura & Empleado & Tiene pareja \\
\hline Inf11 & $\mathrm{H}$ & Heterosexual & 20 & Guadalajara & Preparatoria & Estudiante & Soltero \\
\hline Inf12 & $\mathrm{H}$ & Heterosexual & 35 & Guadalajara & Doctorado & Empleado & Casado \\
\hline Inf13 & $\mathrm{H}$ & Heterosexual & 43 & Guanajuato & Ingeniería & Empleado & Soltero \\
\hline Inf14 & $\mathrm{H}$ & Heterosexual & 30 & Monterrey & Licenciatura & $\begin{array}{l}\text { Negocio } \\
\text { propio }\end{array}$ & Soltero \\
\hline Inf15 & $\mathrm{H}$ & Heterosexual & 28 & CDMX & Licenciatura & Empleado & Tiene pareja \\
\hline Inf16 & $\mathrm{H}$ & Homosexual & 40 & Cd. Juárez & Maestría & Empleado & Tiene pareja \\
\hline
\end{tabular}

Fuente: Elaboración propia.

\section{Panorama sobre las personas childfree en México}

Tal como se argumentó anteriormente, en México la adultez, la masculinidad y la feminidad hegemónicas continúan estando fuertemente ligadas a los roles derivados del modelo tradicional de familia que involucra la maternidad/paternidad. Y, como contraparte a estos discursos, los estigmas hacia las personas childfree que resaltaron en las encuestas y serán analizados a detalle en la sección siguiente son: inmadurez, egoísmo, desconocimiento del amor y tener algo esencialmente mal. 
En esta sección se busca sentar las bases para un panorama sobre las personas mexicanas childfree, a partir de las respuestas de las 163 personas encuestadas y las entrevistas en profundidad. Cabe recordar que las encuestas no fueron aplicadas a una muestra estadísticamente representativa, sino en grupos identificados como childfree y fueron respondidas voluntariamente. Por ello, sólo se pretende identificar tendencias, no hacer una generalización acerca de estas personas.

Aunque en la categoría de sexo se dio la opción de no responder, todos los participantes se identificaron como hombres o mujeres, de acuerdo con la distribución que se muestra a continuación (Tabla 2).

Tabla 2. Participantes en encuesta por sexo y rango de edad

\begin{tabular}{|l|l|l|l|l|l|}
\hline Sexo / rango de edad & $\mathbf{1 8}$ a 29 & $\mathbf{3 0}$ a 39 & $\mathbf{4 0}$ a 49 & $\mathbf{5 0}$ o más & Total \\
\hline Mujer & 37 & 79 & 14 & 0 & 130 \\
\hline Hombre & 12 & 19 & 7 & 0 & 38 \\
\hline Total & 49 & 98 & 21 & 0 & 168 \\
\hline
\end{tabular}

Fuente: Elaboración propia.

Es decir, el 70.8\% corresponde a personas mayores de 29 años, lo cual implica una edad adulta plena en la cual los individuos se han enfrentado a distintas experiencias. Este porcentaje indica que ser childfree no es una etapa propia de la juventud — con toda la ambigüedad del términoni tiene que ver con un reloj biológico; por el contrario, es una decisión pensada y reiterada en la adultez plena.

El 77.4\% de quienes respondieron fueron mujeres. A partir del análisis de la información de las encuestas, se detectan tres motivos por los cuales hubo tanta disparidad con respecto a los varones participantes. El primero, sencillamente porque la participación de las mujeres en los grupos en donde se distribuyó la encuesta es mayor. Esto está ligado a los otros dos: son más mujeres las que están haciendo la elección consciente y definitiva de no tener hijos y, como se argumentará en la siguiente sección, las mujeres mexicanas enfrentan más estigmas que los hombres por su decisión, por lo que buscan crear comunidad y desahogarse con personas que compartan su punto de vista. 
En cuanto a su orientación sexual, los y las participantes se identificaron como sigue: $82.7 \%$ heterosexual, $8.3 \%$ bisexual, $5.4 \%$ homosexual, $1.2 \%$ asexual, $1.2 \%$ otra orientación y el $1.2 \%$ prefirió no responder. El 53\% declaró tener una relación sexo-afectiva en ese momento. Esto reta en números otros de los estigmas que se suelen imponer a las personas childfree en México —el no ser heterosexual y el no ser capaz de conseguir pareja. Como se puede observar, la elección de no tener hijos no tiene relación con las preferencias sexuales ni con el deseo o habilidad para conseguir pareja. Por el contrario, de acuerdo con lo expresado por la mayoría en las encuestas, el estar en pareja es un deseo común y una experiencia que prácticamente todos han tenido.

Por otro lado, consistente con estudios sobre childfree en otras partes del mundo, el $96 \%$ de los encuestados vive en una zona urbana y el $93.4 \%$ dijo tener estudios de licenciatura o posgrado (ver Tabla 3). Es decir, puede advertirse una tendencia mayor entre las personas con mayor nivel educativo a elegir no convertirse en padre o madre. De acuerdo con el análisis, puede argumentarse que, conforme tienen acceso a más educación, las personas tienden a cuestionarse más los discursos hegemónicos y los roles tradicionales y, aunque siguen sufriendo los estigmas, desarrollan la habilidad para desafiarlos e ir encontrando la forma de construirse coherentemente.

Tabla 3. Nivel educativo de participantes por sexo

\begin{tabular}{|l|l|l|l|}
\hline Nivel máximo de educación / sexo & Hombre & Mujer & Total \\
\hline Secundaria & 1 & 0 & 1 \\
\hline Preparatoria & 1 & 9 & 10 \\
\hline Licenciatura & 21 & 67 & 88 \\
\hline Maestría o especialidad & 12 & 43 & 55 \\
\hline Doctorado & 3 & 11 & 14 \\
\hline Total & 38 & 130 & 168 \\
\hline
\end{tabular}

Fuente: Elaboración propia.

Asimismo, el $83.3 \%$ respondió tener empleo o negocio propio, el 12.5\% dedicarse a estudiar de tiempo completo y sólo el $4.2 \%$ dijo ser desempleado. Esto es relevante puesto que, en las encuestas y entrevistas, fue reiterado el comentario de que la situación económica relativamente estable que habían conseguido se vería amenazada en caso de tener hijos, argumentando que los costos asociados a la manutención y crianza los llevarían a un estrato económico más precario 
del actual y eso también impactaría en la calidad de vida de los hijos hipotéticos. En otras palabras, estas personas analizan de forma práctica y realista su capacidad de proveer lo necesario por los años que implica criar a un ser humano, contrario al estigma de egoísmo que se les suele imponer.

Las tendencias en las características de crianza también fueron interesantes. En la Gráfica 1 se puede observar que la mayoría de las personas crecieron en familias tradicionales o con presencia de familiares que representaban arraigados roles paterno y materno. $\mathrm{Y}$, acorde a lo que se identificó en las entrevistas, la decisión de ser childfree no tiene relación con tener modelos de maternidad o paternidad insatisfechos o victimizados. Por el contrario, destacó el hecho de que la mayoría dijo haber tenido madres y padres o familiares cercanos que en su niñez percibían como felices y realizados. Esto contradice el estigma relacionado con traumas de la infancia.

\section{Gráfica 1. Tipo de familia de crianza}

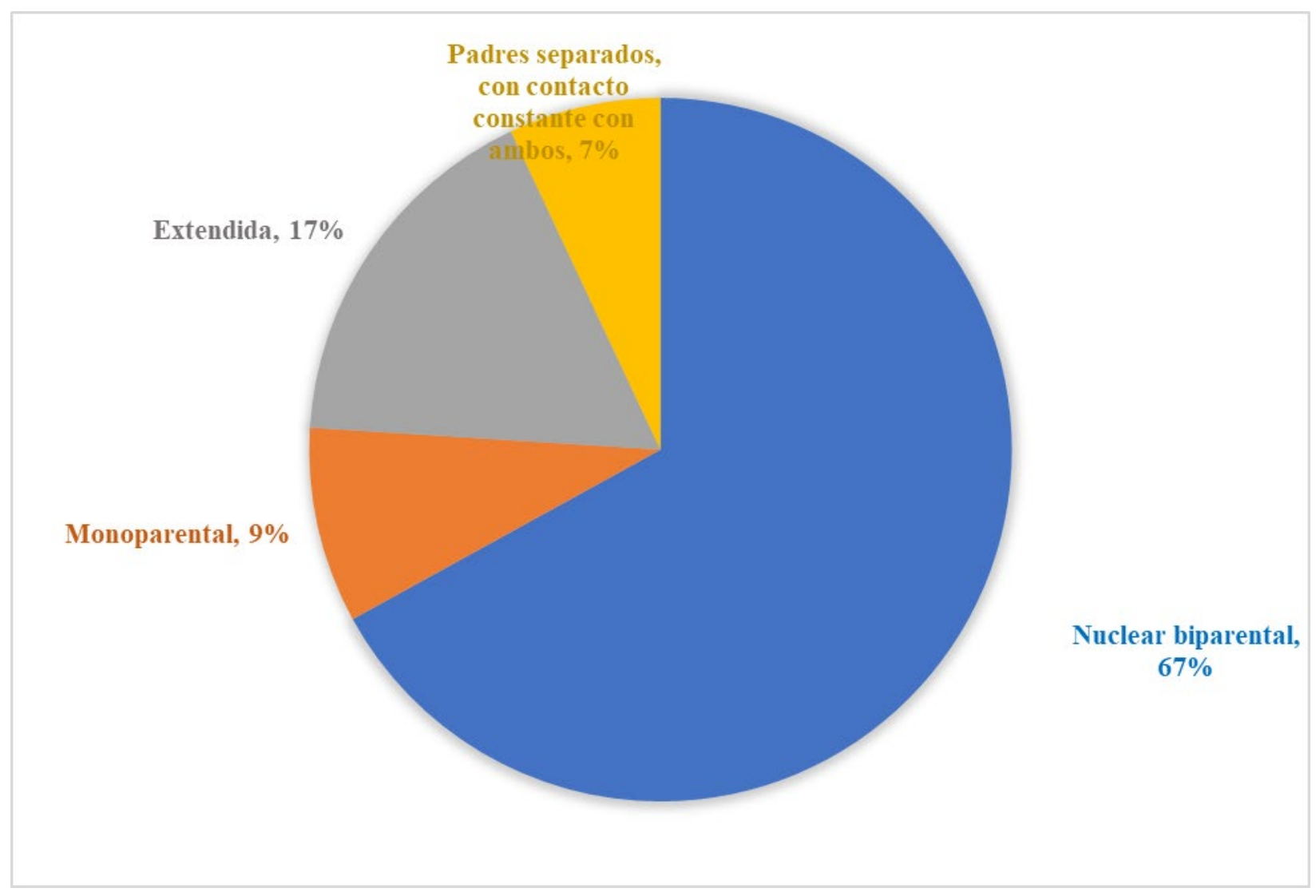

Fuente: Elaboración propia. 
Finalmente, otra característica relevante fue la relacionada con las creencias religiosas. El 86.9\% dijo haber sido inculcado en la infancia con creencias católicas $(80.4 \%)$ o protestantes $(6.5 \%)$. No obstante, en la actualidad sólo el 25\% dijo profesar el catolicismo y el 3\% el protestantismo, con un $64.3 \%$ del total declarando no tener afiliación religiosa. Este elemento se pudo observar también en las entrevistas en profundidad. Debido, entre otras cosas, a que los discursos dominantes en las religiones católica y protestantes ponen tanto énfasis en la maternidad y paternidad como funciones envueltas en designios divinos - relacionadas al milagro de dar vida - la mayoría de las personas childfree encuentran incongruencias entre su decisión y los discursos religiosos.

\section{La deconstrucción de los estigmas}

Las personas childfree son aquellas que toman la decisión de no tener hijos biológicos. De acuerdo con las encuestas y entrevistas, las razones detrás de esta decisión cayeron en las siguientes categorías: la violencia y pobreza estructural del país; la crisis ambiental y la sobrepoblación mundial; no contar con los medios económicos o el tiempo para criar a un ser humano; no tener gusto o paciencia hacia los infantes; considerar que, por condiciones propias — como el hecho de no ser heterosexual, de tener enfermedades hereditarias, etcétera — sus hijos sufrirían estigmas o dificultades. Cabe destacar que la mayoría de las personas dieron más de una razón. Una de las informantes lo indicó así:

En primer lugar, no tengo paciencia con los niños. No los odio, como la gente piensa cuando digo que no quiero, pero no tengo paciencia para cuidarlos 24/7. Creo que, si una persona no está al cien mental, emocional y económicamente, es irresponsable traer a un ser humano al mundo. Y esa es otra. El mundo está en una situación terrible. La contaminación, la sobrepoblación, la crisis económica, la violencia. Si se supone que la gente ama a sus hijos, ¿por qué traerlos a sufrir a este mundo? (Inf8)

Otro expresó lo siguiente: 
No creo que tener hijos sea una necesidad ni algo de instinto, como luego le dicen a uno. Quizá antes era más instintivo, porque la gente no sabía cómo cuidarse, pero ahora tenemos los medios para gozar del sexo sin generar una vida de la cual luego ni se puede hacer uno responsable y que ande ahí con traumas o causando daño. En lo personal, tengo muchos planes, cosas que quiero hacer y sé que me van a consumir tiempo y dinero, no creo que sea justo tener un hijo que no tenga un padre atento a él, y tampoco creo que sea justo que yo sacrifique mis planes por un hijo que no tengo obligación de tener (Inf11).

En la mayoría de los casos, las personas childfree tampoco quieren hijos adoptados. Sin embargo, algunas personas se identifican así porque no quieren procrear, pero no se cierran a la posibilidad de adoptar cuando tengan las condiciones adecuadas, como lo puntualizó una informante en las encuestas:

El deseo de procrear un ser humano nunca ha sido de mi particular interés, además de tener problemas como dismorfia corporal y trastornos alimenticios, por lo tanto, el embarazo no me parece una opción viable. Sin embargo, el cuidado de otros sí es algo que me gustaría hacer. Por esta razón creo que, de tener las condiciones adecuadas en algún momento de mi vida, me gustaría adoptar.

Tal como se ha presentado, dentro de la sociedad mexicana contemporánea el procrear y asumir el rol de madre o padre se promueve como algo natural y sigue siendo uno de los principales marcadores tanto de la adultez normativa, como de la masculinidad y la feminidad hegemónicas. Ante esto, las personas que rechazan dichos roles suelen enfrentarse constantemente a comentarios y cuestionamientos no sólo por parte de su círculo cercano, sino también de los discursos dominantes y las representaciones mediáticas que han conseguido estereotipar a las personas childfree, reduciendo su decisión a una moda o una etapa de la que eventualmente saldrán. 
En esta sección se presentará el análisis a partir de estos estigmas recurrentes que surgieron en las narrativas personales de los informantes, así como de sus impactos en distintos aspectos de su vida cotidiana y los elementos discursivos que utilizan para rechazarlo y reafirmarse a sí mismos.

La decisión de no tener hijos parece ser relativamente respetada por la sociedad mexicana mientras se trate de personas jóvenes. Sin embargo, conforme una persona va dejando esa ambigua barrera de la juventud, las presiones, cuestionamientos y críticas comienzan a aparecer constantemente ligando la decisión a la inmadurez o el egoísmo que se permiten y justifican socialmente en la juventud, pero que resultan indeseables en la etapa de adultez plena. El estigma de la inmadurez parece tener su mayor impacto en los ámbitos laborales y profesionales en el caso de los varones y en el ámbito personal en las mujeres.

Uno de los informantes varones ( Inf10) narró que en alguna ocasión no había sido considerado para un ascenso por ser visto como una persona informal y de fácil movilidad. Otro (Inf13) expresó que sus colegas le hacían comentarios acerca de que sus logros profesionales se debían sólo a que no tenía hijos y, por tanto, podía descansar y rendir mejor en el trabajo. Uno más (Inf15) relató que en su trabajo menospreciaban su vida personal porque, si no tenía hijos, implicaba que no tenía nada importante que hacer fuera del trabajo. Varios más se quejaron de no recibir ciertos bonos o aumentos salariales que sus compañeros con hijos sí recibían, independientemente de su desempeño. Uno de los informantes de las encuestas lo puntualizó así:

En las entrevistas de trabajo siempre me han preguntado que por qué sigo soltero, que por qué no tengo una relación. En algunos casos me hacen otra pregunta, me da demasiada risa: ¿eres gay? Y pues, riéndome, les digo que no. Y, después de compartir puntos de vista sobre esta situación, me han dejado saber que eso lo toman como si fuera una persona que no le gusta comprometerse, entonces eso lo asocian con irresponsable o algo por el estilo. Quiero comentar algo extra, después de haber notado eso en las entrevistas de trabajo que en algún momento tuve, hice un experimento. Tuve como cinco entrevistas y en las cinco dije que estaba en una relación, que no estaba casado, pero sí tenía novia y que estábamos en planes para 
casarnos y que deseaba tener dos hijos y crear una familia y lo gracioso es que en esas cinco entrevistas me ofrecieron empleo.

Es interesante que este estigma de inmadurez en los varones suele ir ligado a un estigma de no ser heterosexual lo cual, en conjunto, tiene un impacto más negativo hacia ellos debido a que en México aún domina un rechazo a las diversidades sexuales. Un informante en entrevista en profundidad lo expresó así:

Entre compañeros de trabajo y jefes siempre ha habido un juicio a mi sexualidad porque no tengo hijos, incluso aunque soy casado. Bromean diciendo que si es pantalla que porque tengo ya ocho años casado y nada de hijos. Lo malo es que no son sólo las bromas, cuando ha habido oportunidades de tomar puestos importantes en mi trabajo y, aunque yo exprese mi interés, ni siquiera me toman en serio y me lo han dicho, entre broma y broma, que yo no tengo la imagen para un director o que yo para qué quiero más dinero si no mantengo hijos y mi esposa trabaja. No me dan una razón relacionada a mi capacidad o experiencia (Inf12).

Aun así, los hombres childfree rechazan el estigma y se afirman como maduros y responsables, aunque eso luego les genere otras consecuencias poco deseables. Uno de ellos indicó:

Mira, yo siempre he trabajado muy duro y me harté de la discriminación siendo empleado, por eso puse mi propio negocio y me va muy bien. Realmente no me importa si piensan que soy gay o que soy irresponsable, pero luego me cargaban la mano con trabajo extra que porque si no tenía familia me podía quedar a cubrir turnos. Cuando me di cuenta de lo ilógico de eso, que te creen irresponsable, pero te dejan más trabajo, decidí que mejor trabajaría para mí mismo (Inf14).

La asignación de trabajo extra a las personas que deciden ser childfree revela un menosprecio a su vida y tiempo privado y es algo que no sólo enfrentan los varones. Las mujeres también lo viven, aunque no relacionado con el estigma de inmadurez, pues, contrario a los varones, el 
estereotipo de las mujeres childfree es que son dedicadas a su trabajo. Por ello, el estigma tiene más efectos en su vida privada. Una informante lo expuso así:

Pues ya sabes, siempre cargan la mano a las que no tenemos hijos. Creo que es discriminación porque a las señoras les dan permiso de salir cualquier cantidad de veces y faltar porque el niño está enfermo, que porque el festival de no sé qué, etcétera. Y, claro, ni hablemos de los beneficios que tienen que nosotras no. Bonos por parto, bonos y festejos y días libres por el día de las madres. Uno que me da mucho coraje es que pueden tener a todos sus hijos en el servicio médico y yo no tengo derecho a un solo beneficiario. Me parece muy injusto cuando aporto lo mismo y termino trabajando más. [...] Ah, sí. Siempre ha sido un problema con parejas y con hombres que he salido. Aunque siempre he sido muy directa desde el principio expresando que no quiero hijos, siempre piensan que voy a cambiar de opinión y tratan de convencerme, pero cuando se dan cuenta que no doy mi brazo a torcer, me acusan de inmadura, egoísta, ya te la sabes. Así que, pues, mejor soltera.

En México, las mujeres que rechazan la maternidad no sólo enfrentan dificultades en el contexto social y familiar, sino que también tienen que enfrentar restricciones al momento de elegir sobre sus propios cuerpos y salud reproductiva debido al estigma de inmadurez. Una informante ( $\operatorname{lnf} 2)$ ha intentado que le realicen un procedimiento de esterilización - salpingoclasia - desde que tenía 22 años. Los diversos ginecólogos a los que ha solicitado el procedimiento, en diferentes momentos de su vida, se lo han negado argumentando ya sea su juventud, que no tiene al menos un hijo o que no tiene un marido que le dé permiso. Ella, ya habiendo superado los 35 años que le pusieron como pretexto la última vez, expresó su frustración con las siguientes palabras: "Ni siquiera cuando quieres puedes porque necesitas permiso de un marido que ni siquiera tienes".

Las distintas informantes que expresaron haber intentado hacerse un procedimiento definitivo para no embarazarse, independientemente de su edad, tuvieron los mismos problemas y recibieron el mismo tipo de argumentos relacionados con su juventud y la posibilidad de cambio de opinión cuando encontraran pareja. Lo anterior lleva implícito el estigma de inmadurez, de 
una supuesta incapacidad de la mujer para decidir sobre su propio cuerpo y trayectoria de vida ante la idea de que el amor puede hacerla cambiar su decisión.

Una constante en las narrativas de las informantes — no así en el caso de los hombres- fue el estigma relacionado con desconocer el amor, el cual se presenta de diversas formas. Una de las más comunes tiene que ver con comentarios que reflejan la idea de que no han conocido a la pareja indicada, dejando implícito que ésta sería quien las convenza de tener hijos. Esta idea es parte clave del estigma y afecta de manera importante a las mujeres childfree, por un lado, porque cuestiona su autonomía y capacidad de decisión; por otro, porque sus parejas lo utilizan como argumento para acusarlas de no amarlos lo suficiente. Todas las informantes, independientemente de su orientación sexual, expresaron haber tenido al menos una relación romántico-afectiva que terminó debido a que sus parejas, que no eran childfree, no tomaron seriamente la decisión expresada por ellas desde el inicio y, conforme avanzaba la relación y ellas no cambiaban de opinión, sus parejas las acusaban de no amarlos. Esta situación fue percibida por la mayoría de ellas como una imposición de culpa ante la ruptura. Una informante lo narró así:

Ahí conoces el tipo de persona que era [tu pareja]. Yo siempre pensé que él no era machista y que me respetaba. Él sabía que yo no quería hijos, se lo dije desde el inicio, pero luego de dos años de relación, me propuso matrimonio. Yo acepté, pero entonces empezó a hacer muchos comentarios sobre hijos y, cuando yo le decía que no iba a tener hijos, me preguntaba que si no lo amaba. Las discusiones fueron escalando hasta que tronamos. Pero en la última, cuando se puso feo, me dijo muchas cosas, que una mujer que no da hijos no sirve, que yo no lo amaba, que seguramente en unos meses estaría embarazada de otro. Ahí me di cuenta de que realmente no era el hombre que pensaba, así que ya cuando se me pasó el dolor y el coraje, me di cuenta de que fue muy bueno todo lo que pasé, pues ningún hombre me iba a convencer de hacer algo que yo no quiera (Inf4).

Otra informante también uso ese tipo de experiencias para reforzar su identidad y decisión: 
Yo me casé pasados los 40 años y sólo porque mi esposo tampoco quiere hijos. Ningún hombre me hizo cambiar de opinión sobre esto, por mucho que estuviera enamorada. Pero eso no lo entiende una sociedad patriarcal como la nuestra (Inf8).

Otra de las formas del estigma del desconocimiento del amor es cuando se une al del egoísmo en el mito de que el verdadero amor desinteresado se conoce sólo por medio de la maternidad/paternidad. Sin embargo, aunque fue mencionado casualmente por un par de informantes varones, no pareció causarles mayores problemas. En cambio, en las mujeres sí fue un tema recurrente en sus narrativas. Las mujeres childfree son acusadas, principalmente por familiares y amistades cercanas, de ser incapaces de amar desinteresadamente. En este sentido, sobre todo aquellas que tienen mascotas o cuidan a familiares, dicen sentir que sus sentimientos y su capacidad de entrega y cuidado desinteresado son minimizados por las personas que les hacen esos comentarios. Así lo expresó una de las informantes:

Amo a mis perros y hago todo porque ellos estén bien, sin importar lo que cueste. Además, hago donaciones a diferentes asociaciones. Pero para esa gente, aparentemente, amor desinteresado es traer otros seres humanos a sufrir pobreza y violencia que cuidar de los seres que ya existen aquí. Mis perros no me van a cuidar cuando esté vieja o enferma, no lo hago por eso, ni por tener un "miniyo", ni por conseguir permisos en el trabajo. Sí me molesta mucho cuando recibo comentarios que menosprecian mi amor por mis mascotas, por mi familia, por mis proyectos. Pero yo sé que son esas personas las que se están perdiendo de conocer otros tipos de amor que son verdaderamente desinteresados (Inf6).

Finalmente, está el estigma de tener algo esencialmente mal como para rechazar la maternidad/paternidad. En este estigma — común en hombres y mujeres-destacó, por un lado, la hipótesis de traumas de la infancia que se achacan a una supuesta falta de modelos maternales/paternales adecuados, a que sufrieron la ruptura de su familia o, incluso, al abuso físico. "Una novia, recién empezaba con ella, cuando le dije que no quería hijos, me preguntó si mis papás eran divorciados" (Inf15). "En el trabajo mencioné una vez ser childfree. Días después, 
andaba el rumor de que habían abusado de mí siendo niña, iy nada que ver! Mi infancia fue muy feliz" (Inf4).

Por otro lado, este estigma puede relacionarse con una acusación a la falta de valores o el odio a la humanidad, mismos que son relacionados comúnmente a no seguir las creencias religiosas cristianas. Una importante proporción de informantes que declararon haber sido criados en hogares católicos o protestantes y ya no profesar esas religiones, mencionaron que sus familias y conocidos solían acusarlos de que su falta de fe —en el discurso social - implicaba una pérdida de valores que, por alguna razón, ligaban directamente a su rechazo a la maternidad/paternidad. Una de las informantes relató al respecto:

Yo soy de familia tradicional y muy católica. Ya te imaginarás cuando me di cuenta de que era bisexual. Primero traté de ocultarlo, luego decidí hablar con mi pastor de confirmaciones, porque yo sí era creyente, pero me dijo que eso no era lo que Dios quería y que, si me gustaban los hombres, simplemente evitara relacionarme con mujeres. Pero, justo en ese tiempo, yo estaba enamorada de una chica y, luego de pensarlo mucho, me di cuenta de que, si en la iglesia no me aceptaban así, entonces no era cierto todo lo que decían y me alejé. Cuando, unos años después, les dije a mis papás que no quería tener hijos, me echaron todo en cara. Que mi bisexualidad y no querer hijos era porque había dejado la iglesia y que dónde habían quedado los valores que me habían inculcado. [...]A partir de eso me he alejado mucho de mi familia porque sé que me ven como una mala persona. Así que prefiero mantener mi distancia. Yo sé que mis decisiones no hacen daño y que no soy mala, pero ellos así me ven (Inf5).

Otro informante indicó:

¿Has visto los reportajes que salen cada rato en internet? Dicen que no tener hijos es un movimiento que quiere acabar con la humanidad o que odiamos a los niños. La verdad yo ni odio a los niños ni quiero acabar con la humanidad, aunque creo que deberíamos ser más responsables hacia lo que estamos haciendo al planeta. 
[...]No significa que mi esposa o yo seamos parte de un movimiento para acabar con la raza humana, son decisiones personales. [...]Aunque no lo creas, incluso en la universidad hay colegas que hacen comentarios que me hacen pensar que creen que soy un sociópata $(\operatorname{Inf12})$.

En general, las personas childfree perciben el estigma de tener algo mal, pero están convencidas de que su decisión no las vuelve ni malas ni antinaturales. Contrario a los estigmas mencionados, tanto en las encuestas como en las entrevistas en profundidad, las razones para no tener hijos fueron claramente identificadas y razonadas teniendo en mente no sólo el bienestar propio sino la dificultad que, en el mundo contemporáneo, implica conseguir estabilidad y felicidad para un hijo o hija hipotéticos.

\section{Reflexiones finales}

Debido a la característica exploratoria del estudio, no se pretende llegar a conclusiones generalizadoras, sino aportar, desde México, a la creciente investigación sobre el tema de la elección childfree y visibilizar también las experiencias de los hombres, no sólo de las mujeres. En los casos analizados aquí, los informantes reforzaron narrativamente su decisión e identidad, deconstruyendo los estigmas a partir de sus experiencias. Estas personas childfree mexicanas tienen claramente identificadas las razones por las que no quieren hijos. Tienen en cuenta elementos personales y estructurales que las llevan a tomar una decisión basada no sólo en deseos presentes, sino también en las consecuencias personales, sociales y del hijo hipotético.

No obstante, es innegable que, mientras las ideologías dominantes y los discursos mediáticos continúen colocando a la maternidad y paternidad como transiciones naturales hacia la adultez y a las personas childfree como parte de un movimiento en contra de la humanidad, los individuos que no siguen dichas trayectorias pueden experimentar conflictos al momento de construirse a sí mismos de una forma positiva. Tal como dice Daniluk (1999), las personas necesariamente voltean hacia los discursos dominantes en su sociedad para comprender qué se espera de ellas, qué es normal y para darle sentido a sus sentimientos y comportamientos. Lo anterior es lo que se entiende como hegemonía de género, la cual se produce y reproduce dentro de las sociedades, se justifica y refuerza por medio de la mayoría de las actividades dentro de la cultura, y expande 
dogmas acerca de lo que hace a alguien hombre o mujer, las categorías dicotómicas que son reconocidas como naturales por la heteronormatividad. A pesar de la existencia de diferentes versiones de masculinidad y feminidad, la hegemonía de género se encuentra intrínseca en esas identidades, impone prácticas sociales normativas para varones y mujeres, naturaliza mitos y valores que sirven de mapas para que las personas se localicen dentro de ellos (Bonino, 2002).

Este proceso parece ser más simple en los varones cuando poseen independencia económica o un considerable desarrollo profesional. Puesto que la masculinidad hegemónica en México está esencialmente ligada al rol público del hombre como trabajador y al rol privado de proveedor, los hombres entrevistados, en su mayoría con empleo y económicamente autosuficientes, fueron capaces de ligar estas cualidades a los roles normativos, justifican para sí mismos su masculinidad, independientemente de su orientación sexual.

Por otro lado, consistente con estudios previos enfocados en mujeres childfree alrededor del mundo, las informantes tuvieron que enfrentar más estigmas. En la sociedad mexicana sigue dominando la idea de que es natural que una mujer sienta la necesidad y el deseo de convertirse en madre, pues no tener un hijo es visto como la imposibilidad de realizarse como mujer; además, rechazar la maternidad se considera generalmente como una expresión de egoísmo, inmadurez y desconocimiento del amor. Bajo la preeminencia de este discurso, las mujeres que deciden no ser madres son socialmente cuestionadas acerca de su normalidad y enfrentan presión tanto en sus círculos privados como laborales.

\section{Referencias bibliográficas}

Aguilar Montes de Oca, Yessica Paola; Valdez Medina, José Luis;, González-Arratia LópezFuentes, Norma Ivonne y González Escobar, Sergio (2013). Los roles de género de los hombres y las mujeres en el México Contemporáneo. Enseñanza e Investigación en Psicología, 18(2), 207-224. 
Ávila González, Yanina. (2005). Mujeres frente a los espejos de la maternidad: las que eligen no ser madres. Desacatos, 17, 107-126. Recuperado de http://www.scielo.org.mx/scielo.php?script=sci_abstract\&pid=S1607050X2005000100007\&lng=es\&nrm=iso

Bartlett, Jane. (1996). Will you be Mother? Women who choose to say no. London: Virago Press.

Berghegger, Scott. (2009). Yo soy yo y mi circunstancia: The Socioeconomic Origins of Machismo and the Macho. Inquiries Journal/Student Pulse, 1(11), 1-7. Recuperado de http://www.inquiriesjournal.com/a?id=27

Bonino, Luis. (2002). Masculinidad hegemónica e identidad masculina. Dossiers feministes 6. Masculinitats: Mites, de/construccions $i$ mascarades, (6) 7-37. Recuperado de http://www.raco.cat/index.php/DossiersFeministes/article/viewFile/102434/153629

de Beauvoir, Simone. (2014). The second sex. En Classic and Contemporary Readings in Sociology (pp. 11- 126).doi: https://doi.org/10.4324/9781315840154

Butler, Judith. (1990). Gender Trouble: Feminism and the Subversion of Identity. New York: Routledge.

Butler, Judith. (2011). Bodies that matter. En Bodies that Matter. On the Discursive Limits of Sex (pp. 1-25). doi: https://doi.org/10.4324/9780203828274

Cardozo-Freeman, Inez. (1975). Games Mexican Girls Play. The Journal of American Folklore, 88(347), 12-24. doi: https://doi.org/10.2307/539182

Chancey, Laurie y Dumais, Susan A. (2009). Voluntary childlessness in Marriage and Family Textbooks, 1950-2000. Journal of Family History, 34(2), 206-223. doi: https://doi.org/10.1177/0363199008330733 
Dales, Laura. (2014). Ohitorisama, Singlehood and Agency in Japan. Asian Studies Review, 38(2), 224-242. doi: https://doi.org/10.1080/10357823.2014.902033

Dales, Laura. (2015). Suitable single?: representations of singlehood in contemporary Japan. En Tomoto Aoyama, Laura Dales y Romit Dasgupta (Eds.), Configurations of Family in Contemporary Japan (pp. 21-32). New York: Routledge.

Díaz Domínguez, Alejandro. (febrero 1 de 2021). ¿Qué nos dice el Censo 2020 sobre religión en México? Nexos. Recuperado de https://datos.nexos.com.mx/que-nos-dice-el-censo-2020sobre-religion-en-mexico/

Foucault, Michel. (2005). Historia de la sexualidad I. La voluntad de saber. México: Siglo XXI editores.

Fraser, Nancy. (1997). Heterosexism, Misrecognition, and Capitalism: A Response to Judith Butler. Social Text, (52-53), 279-289. doi: https://doi.org/10.2307/466745

García Delgado, Elisa y Riquelme Vigueras, Ana María. (2017). Percepción de los roles de género en la cultura mexicana actual. Memorias del Concurso Lasallista de Investigación, Desarrollo e innovación, 4(1), 47- 51. Recuperado de http://revistasinvestigacion.lasalle.mx/index.php/mclidi/article/view/1332

Gillespie, Rosemary. (2003). Childfree and feminine: Understanding the gender identity of voluntarily childless women. Gender and Society, 17(1), 122-136. doi: https://doi.org/10.1177/0891243202238982

Ginsburg, Faye y Rapp, Rayna. (1991). The Politics of Reproduction. Annual Review of Anthorpology, 20, 311-343. doi: https://doi.org/10.1146/annurev.an.20.100191.001523

Goffman, Erving. (2006). Estigma. La identidad deteriorada. Buenos Aires: Amorrortu editores. 
Gonzalez-Barrientos, Marcela y Napolitano, Stefania. (2015). Beyond phallic domain: Female otherness as a resource for liberation: Some notes from lacanian psychoanalysis and poststructuralist feminism. The Psychoanalytic Review, 102(3). doi: https://doi.org/10.1521/prev.2015.102.3.365

Gutmann, Matthew. (2000). Ser hombre de verdad en la Ciudad de México. Ni macho ni mandilón. México: El Colegio de México.

Hammack, Phillip L. (2008). Narrative and the cultural psychology of identity. Personality and Social Psychology Review, 12(3), 222-247. doi: https://doi.org/10.1177/1088868308316892

Instituto Nacional de Estadística y Geografía. (2018). "Estadísticas a propósito del Día de la madre (10 de mayo)". https://www.inegi.org.mx/contenidos/saladeprensa/aproposito/2018/madre2018_Nal.pdf

Instituto Nacional de Estadística y Geografía. (2021). Nota Técnica. Estadísticas a propósito del 14 de febrero. https://www.inegi.org.mx/contenidos/saladeprensa/aproposito/2021/EAP_14FEB21.pdf

Instituto Nacional de Estudios Históricos de las Revoluciones de México. (2015). Ley de Matrimonio Civil. Constitución $1917 . \quad$ Recuperado de https://constitucion1917.gob.mx/es/Constitucion1917/Ley_de_Matrimonio_Civil

Instituto Nacional de las Mujeres. (2007). El impacto de los estereotipos y los roles de género en México. 16. Recuperado de http://cedoc.inmujeres.gob.mx/documentos_download/100893.pdf

Jones, Ann Rosalind. (1984). Julia Kristeva on Femininity: The Limits of a Semiotic Politics. Feminist Review 18(1), 56-73. doi: https://doi.org/10.2307/1394859 
Link, Bruce G. yPhelan, Jo C. (2001). Conceptualizing Stigma. Annual Review of Sociology, 27, 363-385. doi: https://doi.org/10.1146/annurev.soc.27.1.363

MacInnes, John y Pérez Díaz, Julio. (2009). The reproductive revolution. The Sociological Review, 57(2), 262-284. doi: https://doi.org/10.1111/j.1467-954X.2009.01829.x

Mandujano-Salazar, Yunuen Ysela. (2017). It is Not that I Can't, It is that I Won't: The Struggle of Japanese Women to Redefine Female Singlehood through Television Dramas. Asian Studies Review, 41(4), 526-543. doi: https://doi.org/10.1080/10357823.2017.1371113

Mandujano-Salazar, Yunuen Ysela. (2019). Exploring the Construction of Adulthood and Gender Identity Among Single Childfree People in Mexico and Japan. SAGE Open, 9(2). 112. doi: https://doi.org/10.1177/2158244019855844

McCabe, Katherine y Sumerau, J. E. (2018). Reproductive Vocabularies: Interrogating Intersections of Reproduction, Sexualities, and Religion among U.S. Cisgender College Women. Sex Roles, 78(5-6), 352-366. doi: https://doi.org/10.1007/s11199-017-0795-2

Morgan, Lynn M. y Roberts, Elizabeth. (2012). Reproductive governance in Latin America. Anthropology \& Medicine, 19(2), 241-254. doi: https://doi.org/10.1080/13648470.2012.675046

Nishi, F., Kan, M. (2006). Current Situation of Parasite-singles in Japan (Summary). http://www.stat.go.jp/training/english/reseach/parasite_eng.pdf

Petchesky, Rosalind P. (1990). Abortion and woman's choice: the state, sexuality, and reproductive freedom. Mishawaka: Northeastern University Press. Recuperado de http://hdl.handle.net/2027/heb.00493.0001.001

Peterson, Helen. (2014). Fifty shades of freedom. Voluntary childlessness as women's ultimate liberation. Women's Studies International Forum. 53, 182-191. doi: 
https://doi.org/10.1016/j.wsif.2014.10.017

Quintal López, Rocío. (2002). La presión social hacia mujeres que desafían el paradigma "mujer igual a madre". GénEros, 9(27), 42-49. doi: http://bvirtual.ucol.mx/descargables/548_presion_social.pdf

Rankin, Monica A. (2011). Mexicanas en guerra: World War II and the Discourse of Mexican Female Identity. Frontiers: A Journal of Women Studies, 32(2), 83-110. doi: https://doi.org/10.5250/fronjwomestud.32.2.0083

Robertson, John A. (1994). Children of Choice: Freedom and the New Reproductive Technologies. Princeton: Princeton University Press.

Rojas Martínez, Olga Lorena. (2012). Masculinidad y vida conyugal en México. Cambios y persistencias. GénEros, $18(10), \quad 79-104 . \quad$ doi: http://bvirtual.ucol.mx/descargables/378_masculinidad_vida_conyugal.pdf

Rosenberger, Nancy. (2007). Rethinking Emerging Adulthood in Japan: Perspectives From Long-Term Single Women. Child Development Perspectives 1(2), 92-95. doi: https://doi.org/10.1111/j.1750-8606.2007.00021.x

Ruiz-Alfaro, Sofia. (2018). A Threat to the Nation: "México marimacho" and Female Masculinities in Postrevolutionary Mexico. Hispanic Review, 81(1), 41-62. doi: http://www.jstor.org/stable/43278931

Salyakhieva, Liliya Maratovna y Saveleva, Zhanna Vladimirovna. (2017). Childfree as a Social Phenomenon: Russians' Attitude to Voluntary Childlessness. Journal of History Culture and Art Research, 6(4), 531-537. doi https://doi.org/10.7596/taksad.v6i4.1144

Schell, Patience A. (2012). Género, clase y ansiedad en la Escuela Vocacional Gabriela Mistral, revolucionaria Ciudad de México. En Gabriela Cano, Mary Kay Vaughan y Jocelyn Olcott 
(Eds.), Género, poder y política en el México posrevolucionario (pp. 173-195). Fondo de Cultura Económica.

Yamada, Masahiro. (1999). Parasaito Shinguru no Jidai. Chikuma Shobo.

Yeshua-Katz, Daphna. (2018). Blame or Shame: The Search for Online Support and Stigma Origin Among Israeli Childless Women. Mass Communication and Society, 22, 117-138. doi: https://doi.org/10.1080/15205436.2018.1469152

\section{YUNUEN YSELA MANDUJANO-SALAZAR}

Es profesora investigadora del Departamento de Ciencias Sociales de la Universidad Autónoma de Ciudad Juárez. Tiene licenciatura en Economía, maestría en Estudios de Asia y África especialidad Japón y doctorado en Ciencias Sociales. Es miembro del Sistema Nacional de Investigadores Nivel 1. Sus líneas de investigación son: identidades y vida cotidiana; discursos hegemónicos y medios de comunicación; estudios japoneses. 